of PPODA-QT tothe positive controls results in statistically significant reduction of protein depletion from blood samples.Verification of this result via analysis of desorbed proteins is underway.

Conclusion The protein-resistance of PPODA-QT as shown in this study makes it an interesting material candidatefor a variety of surgical applications. A liquid-to-solid curing material with inherent protein-resistantproperties could be utilized not only as a novel liquid embolic for treatment of intracranial aneurysmsand AVMs, but could also be used as a non-fouling, bioinert coating for metallic implants such as stents,flow diverters, and coils.

Disclosures W. Merritt: 1; C; NIH STTR Phase II (\#1R41NS097069-01A1). 2; C; Aneuvas Technologies, Inc. A. Koppisch: None. R. Kellar: None. A. Ducruet: 4; C; Aneuvas Technologies, Inc. T. Becker: 4; C; Aneuvas Technologies, Inc.

\section{E-011 IN VITRO AND IN VIVO EXPERIMENTS OF A NOVEL INTRA-ARTERIAL NEUROVASCULAR DECOMPRESSOR FOR TREATING NEUROVASCULAR COMPRESSION SYNDROMES}

J Chung*. Department of Neurosurgery, Severance Hospital, Yonsei Univeristy College of Medicine, Seoul, Korea, Republic of

\subsection{6/neurintsurg-2020-SNIS.47}

Objectives Neurovascular compression syndromes (NVCS) could be cured with an intravascular device that releases compression of the root entry zone of cranial nerves by changing the course of offending vessels. The purpose of this study was to report our results of in vitro and in vivo experiments with a novel intra-arterial neurovascular decompressor (IA-NVD) for NVCS.

Methods A nitinol-based IA-NVD was developed to release pressure applied to the root entry zone of cranial nerves by changing the course or angle of an offending vessel, which can possibly cure NVCS. We performed in vitro tests for safety and feasibility and preliminary in vivo tests up to 4 weeks for safety.

Results The bending stiffness of the device was similar to but slightly stronger than that of current, closed-cell intracranial stents. Hemocompatibility tests showed no significant thrombogenesis in whole blood. After the 4-week follow-up, all animals (20-month-old female Gottingen mini-pigs weighing 15$18 \mathrm{~kg}, \mathrm{n}=4$ ) had a normal upright position and gait. Scanning electron microscopy images and H\&E staining of arteries containing the devices showed good neointima formation on the devices. Intima hyperplasia occurred over wires and connecting tubes, but it did not interrupt the patency of the arterial lumen.

Conclusion An IA-NVD was created and tested to demonstrate its functionality and biocompatibility in the present experiments. The device may be safely applied to intracranial arteries, providing us a chance to test the efficacy of an upgrade version of the device on changing the course of an artery that compresses a cranial nerve.

Disclosures J. Chung: None.

\section{E-012 ENDOVASCULAR BIOPSY OF VERTEBROBASILAR ANEURYSM DEMONSTRATES DIFFERENTIAL EXPRESSION OF IMMUNOLOGIC GENES IN POLYARTERITIS NODOSA PATIENT}

K Narsinh* ${ }^{*}$ K Narsinh, D McCoy, Z Sun, E Winkler, A Abla, D Cooke. UCSF, San Francisco, $C A$

10.1136/neurintsurg-2020-SNIS.48

Introduction Personalized medicine refers to the practice of tailoring intervention to an individual based on their unique genotypic or phenotypic characteristics. Gene expression

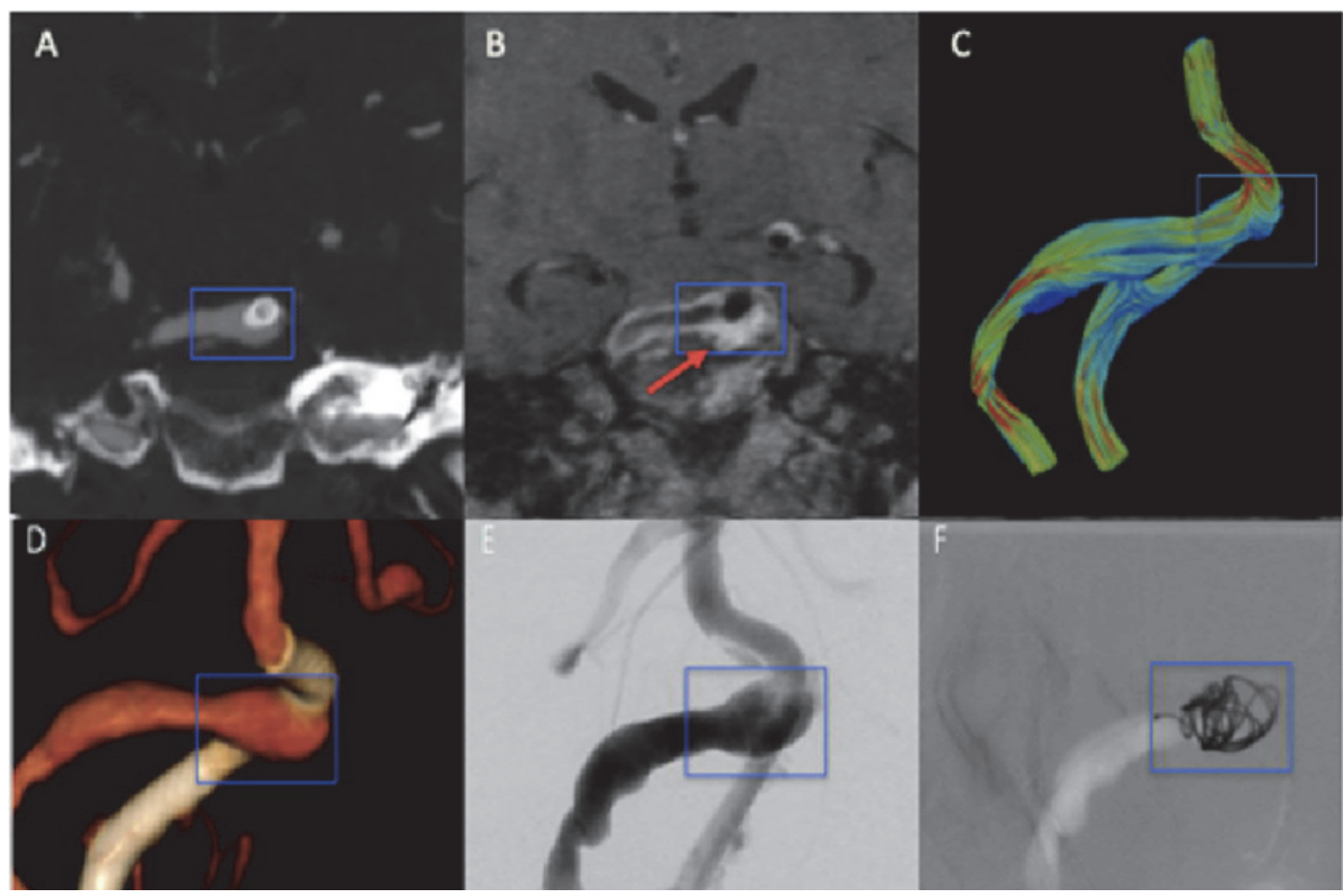

